\title{
OSCA: a tool for omic-data-based complex trait analysis
}

\author{
Futao Zhang ${ }^{1}$, Wenhan Chen', Zhihong Zhu', Qian Zhang ${ }^{1}$, Marta F. Nabais ${ }^{1,2}$, Ting Qi ${ }^{1}$, lan J. Deary ${ }^{3}$, \\ Naomi R. Wray ${ }^{1,4}$, Peter M. Visscher ${ }^{1,4}$, Allan F. McRae ${ }^{1}$ and Jian Yang ${ }^{1,4,5^{*}}$ (D)
}

\begin{abstract}
The rapid increase of omic data has greatly facilitated the investigation of associations between omic profiles such as DNA methylation (DNAm) and complex traits in large cohorts. Here, we propose a mixed-linear-model-based method called MOMENT that tests for association between a DNAm probe and trait with all other distal probes fitted in multiple random-effect components to account for unobserved confounders. We demonstrate by simulations that MOMENT shows a lower false positive rate and more robustness than existing methods. MOMENT has been implemented in a versatile software package called OSCA together with a number of other implementations for omic-data-based analyses.
\end{abstract}

\section{Introduction}

The rapid proliferation of genetic and omic data in large cohort-based samples in the past decade has greatly advanced our understanding of the genetic architecture of omic profiles and the molecular mechanisms underpinning the genetic variation of human complex traits [1-3]. These advances include the identification of a large number of genetic variants associated with gene expression [4, 5], DNA methylation [6,7], histone modification $[8,9]$, and protein abundance [10,11]; the discovery of omic measures associated with complex traits $[12,13]$; the improved accuracy in predicting a trait using omic data [14, 15]; and the prioritization of gene targets for complex traits by integrating genetic and omic data in large samples $[3,13,16-18]$. These advances have also led to the development of software tools, focusing on a range of different aspects of omic data analysis. Therefore, a software tool that implements reliable and robust statistical methods for comprehensive analysis of omic data with high-performance computing efficiency is required.

A well-recognized challenge in omic-data-based analysis is to control for false positive rate (FPR) in the presence of confounding factors, as failing to model the confounders

\footnotetext{
* Correspondence: jian.yang.qt@gmail.com

${ }^{1}$ Institute for Molecular Bioscience, The University of Queensland, Brisbane,

Queensland 4072, Australia

${ }^{4}$ Queensland Brain Institute, The University of Queensland, Brisbane,

Queensland 4072, Australia

Full list of author information is available at the end of the article
}

may lead to spurious associations [19-21] and/or a loss of statistical power [22]. While some confounders (e.g., age and sex) are known and available in most data so that their effects can be accounted for by fitting them as covariates in linear models, others are either uncharacterized or difficult to measure. For example, in DNA methylation (DNAm) data from whole blood, cell type compositions (CTCs) are evident confounders in a methylome-wide association study (MWAS; also known as an epigenomewide association study or EWAS) [21, 23, 24] although CTCs may be useful for the prediction of some phenotypes. CTCs tend to be correlated with the DNAm at CpG sites that are differentially methylated in different cell types (namely differentially methylated sites) and have been shown to be associated with age and multiple traits and diseases [19, 21, 25, 26]. MWAS analysis without accounting for CTCs could give rise to biased test statistics unless neither CTCs nor DNAm sites are associated with the trait in question. Although it is possible to measure CTCs directly or predict them by reference-based prediction methods $[27,28]$, reference-free methods that are able to correct for confounding effects without the need of characterizing all the confounders have broader applications [22, 29-32]. Moreover, the predicted CTCs often only explain a certain proportion of variation in CTCs resulting in biased test statistics due to the uncaptured variation in CTCs. Existing reference-free methods are mainly based on the strategy of fitting a number of 
covariates (estimated from factor analysis or similar approaches with or without reference $[22,29,31,32]$ ) in a fixed-effect model or a set of selected DNAm probes in a mixed linear model (MLM) [30]. However, uncharacterized confounders with small to moderate effects and numerous correlations between distal DNAm probes (e.g., those on different chromosomes) induced by the confounders may not be well captured by either a fixed number of principal features or a subset of selected probes.

In this study, we proposed a reference-free method (called MOA: MLM-based omic association) that fits all probes as random effects in an MLM-based association analysis to account for the confounding effects, including the correlations among distal probes induced by the confounding. We then extended the method to stratify the probes into multiple randomeffect components (called MOMENT: multicomponent MLM-based omic association excluding the target) to model a scenario where some probes are much more strongly associated with the phenotype than others. We evaluated the performance of MOA and MOMENT by extensive simulations and demonstrated their reliability and robustness in comparison with existing methods. We have implemented MOA and MOMENT together with a comprehensive set of other methods for omic data analysis in an easy-to-use and computationally efficient software package, OSCA (omic-data-based complex trait analysis).

\section{Results}

\section{Overview of the OSCA software}

OSCA comprises four main modules: (1) data management for which we designed a binary format to efficiently store and manage omic data; (2) linear-regression- and MLM-based methods (including the methods proposed in this study) to test for associations between omic measures and complex traits; (3) methods to estimate the proportion of variance in a complex trait captured by all the measures of one or multiple omic profiles (e.g., all SNPs and DNAm probes) and to predict the trait phenotype in a new sample based on the joint effects of all omic measures estimated in a discovery sample; and (4) an efficient implementation of the methods to identify genetic variants associated with an omic profile, e.g., DNA methylation quantitative trait loci (mQTL) analysis. We will describe the methods based on DNAm data, but the methods and software tool are in principle applicable to other types of omic data including gene expression, histone modification, and protein abundance. The computer code of OSCA is written in $\mathrm{C}++$ programming language and supports multi-threading based on OpenMP for highperformance computing. The compiled binary files are freely available at http://cnsgenomics.com/software/osca/.

\section{MLM-based omic association analysis methods}

One of the primary applications of OSCA is to test for associations between omic measures (e.g., DNAm probes) and a complex trait (e.g., body mass index (BMI)) correcting for confounding effects. In an MWAS, the test statistics of null probes can be inflated because of the associations of probes with confounders that are correlated with the phenotype. Note that, even if the confounders are not directly associated with the phenotype, the presence of confounders (e.g., CTCs or experimental batches) can cause correlations between the traitassociated probes and the null probes in distal genomic regions or even on different chromosomes, giving rise to inflated test statistics of the null probes (see the simulation results below). Existing methods that fit a number of covariates computed from dimension reduction approaches in a fixed-effect model $[22,31,32]$ or a set of selected DNAm probes in an MLM [30] may not be sufficient to correct for confounding effects widely spread among a large number of probes or correlations between distal probes induced by the confounding. We propose two MLM-based approaches (MOA and MOMENT) that include all the (distal) probes as random effects in the model to account for the effects of the confounders on the trait and probes as well as the correlations among distal probes. We show by simulations (see below) that both MOA and MOMENT are more robust than existing methods in controlling for false positive rate (FPR) and family-wise error rate (FWER) in MWAS (see below).

Here, we start with a general MLM that fits all probes as random effects, i.e.,

$$
\mathbf{y}=\mathbf{C} \boldsymbol{\beta}+\mathrm{Wu}+\mathbf{e}
$$

where $\mathbf{y}$ is an $n \times 1$ vector of phenotype values with $n$ being the sample size, $\mathbf{C}$ is an $n \times p$ matrix for covariates (e.g., age and sex) with $p$ being the number of covariates, $\boldsymbol{\beta}$ is a $p \times 1$ vector of the effects of covariates on the phenotype, $\mathbf{W}$ is an $n \times m$ matrix of standardized DNAm measures of all $m$ probes, $\mathbf{u}$ is an $m \times 1$ vector of the joint effects of all probes on the phenotype, and $\mathbf{e}$ is an $n \times 1$ vector of residuals. In this model, $\boldsymbol{\beta}$ are fixed effects whereas $\mathbf{u}$ and $\mathbf{e}$ are random effects with $\mathbf{u} \sim N(\mathbf{0}, \mathbf{I}$ $\left.\sigma_{u}^{2}\right)$ and $\mathbf{e} \sim N\left(\mathbf{0}, \mathbf{I} \sigma_{e}^{2}\right)$. The variance-covariance matrix for $\mathbf{y}$ is $\operatorname{var}(\mathbf{y})=\mathbf{V}=\mathbf{W} \mathbf{W} / \sigma_{u}^{2}+\mathbf{I} \sigma_{e}^{2}$. This equation can be re-written as.

$$
\mathbf{V}=\mathbf{A} \sigma_{o}^{2}+\mathbf{I} \sigma_{e}^{2} \text { with } \mathbf{A}=\mathbf{W} \mathbf{W}^{\prime} / m \text { and } \sigma_{o}^{2}=m \sigma_{u}^{2}
$$

where $\mathbf{A}$ is defined as the omic-data-based relationship matrix (ORM) ("Methods" section) and $\sigma_{o}^{2}$ is the amount of phenotypic variance captured by all probes. The variance components $\left(\sigma_{o}^{2}\right.$ and $\left.\sigma_{e}^{2}\right)$ in such an MLM can be estimated by REML algorithms [33]. Analogous to the method for estimating SNP-based heritability [34, 35], 
the proportion of variance in the phenotype captured by all the probes can be defined as $\rho^{2}=\sigma_{o}^{2} /\left(\sigma_{o}^{2}+\sigma_{e}^{2}\right)$. We name this variance-estimation method OREML following the nomenclature of GREML [34]. The estimated joint probe effects $(\hat{\mathbf{u}})$ from this model by a random-effect estimation approach (e.g., BLUP [36]) can be used to predict the phenotypes of individuals in a new sample based on omic data, i.e., $\hat{\mathbf{y}}_{\text {new }}=\mathbf{W}_{\text {new }} \hat{\mathbf{u}}$. We call this OBLUP.

Model [1] can be extended to test for association between a probe $i$ and the trait, i.e.,

$$
\mathbf{y}=\mathbf{w}_{i} b_{i}+\mathbf{C} \boldsymbol{\beta}+\mathbf{W} \mathbf{u}+\mathbf{e} \text { with } \mathbf{V}=\mathbf{W} \mathbf{W}^{\prime} \sigma_{u}^{2}+\mathbf{I} \sigma_{e}^{2}
$$

In comparison to model [1], this model has two additional terms, $\mathbf{w}_{i}$ (an $n \times 1$ vector of standardized DNAm measures of a probe $i$, i.e., the target probe) and $b_{i}$ (the effect of probe $i$ on the phenotype; fixed effect). The probe effect $b_{i}$ (together with the covariates' effects) can be estimated by the generalized least squares (GLS) approach, i.e., $\left[\hat{b}_{i} \hat{\boldsymbol{\beta}}\right] \prime=\left(\mathbf{X} / \mathbf{V}^{-1} \mathbf{X}\right)^{-1} \mathbf{X} / \mathbf{V}^{-1} \mathbf{y}$ and $\operatorname{var}\left[\hat{b}_{i} \hat{\boldsymbol{\beta}}\right]^{T}$ $=\left(\mathbf{X} / \mathbf{V}^{-1} \mathbf{X}\right)^{-1}$ with $\mathbf{X}=\left[\mathbf{w}_{i} \mathbf{C}\right]$. The sampling variance (standard error (SE) squared) of $\hat{b}_{i}$ is the first diagonal element of $\operatorname{var}\left[\hat{b}_{i} \hat{\boldsymbol{\beta}}\right]^{T}$. The null hypothesis $\left(H_{0}: b_{i}=0\right)$ can be tested by a two-sided $t$ test (or approximately chi-squared test if sample size is large) given $\hat{b}_{i}$ and its SE. We call this method MOA. Applying this method to test each of the probes across the genome is extremely computationally expensive because the variance components $\sigma_{u}^{2}$ and $\sigma_{e}^{2}$ need to be estimated repeatedly for each probe by REML that requires the computation of $\mathbf{V}^{-1}$ (computational complexity of $O\left(n^{3}\right)$ ) multiple times in an iterative process. To speed up the computation, we use a two-step approach as in [37] to compute $\mathbf{V}^{-1}$, with the first step to perform an eigendecomposition of $\mathbf{W W}$ ' and the second step to compute $\mathbf{V}^{-1}$ based on the eigenvalues and eigenvectors. Since the eigendecomposition only needs to be done once for the whole genome scan, this two-step approach reduces the complexity of computing $\mathbf{V}^{-1}$ by orders of magnitude when testing each specific probe. Moreover, as the proportion of phenotypic variance attributable to a single probe is often very small, we can further speed up the computation by an approximate approach (similar to the approximate MLM-based GWAS methods [38, 39]) that only requires to compute $\mathbf{V}^{-1}$ once, assuming that the estimates of $\sigma_{u}^{2}$ and $\sigma_{e}^{2}$ under the null (i.e., $b_{i}=0$ ) are approximately equal to those under the alternative (i.e., $b_{i} \neq 0$ ). Both the approximate and exact MOA approaches have been implemented in OSCA.

There are two properties of the MOA method worthy of consideration. First, the target probe is fitted twice in the MOA model, once as a fixed effect $\left(b_{i}\right)$ and again as a random effect (the $i$ th element of $\mathbf{u}$ ), resulting in a loss of power to detect $b_{i}$ (a recognized issue in MLM-based association analysis with SNP data $[39,40])$. This problem can be solved by leaving out probes in close physical proximity of the target probe (including the target) from the random-effect term because DNAm status of CpG sites in close physical proximity is likely to be regulated by the same mechanism and therefore tends to be highly correlated. This strategy has been used previously in both GWAS (genome-wide association study) $[39,40]$ and MWAS [30]. In practice, we exclude the probes $<50 \mathrm{~kb}$ from the target probe. Note that the distance parameter may differ for other types of omic data (e.g., a window size of $100 \mathrm{kbp}$ is recommended for gene expression data; see below for details). Second, MOA assumes a single distribution to all the probe effects in the random-effect term, which may not be well fitted to data if some probes have much stronger associations with the trait than other probes. For example, if CTCs are associated with the phenotype, then all the probes that are highly differentially methylated in different cell types [41-43] may present a very different distribution of effects from the other probes. One solution to this issue is to stratify the probes into multiple groups by the association test statistics (from linear regression) and fit them as separate random-effect terms in the model. We extended the MOA method with the two modifications mentioned above and named it as MOMENT (multicomponent MLM-based omic association excluding the target). The MOMENT model can be written as

$$
\mathbf{y}=\mathbf{w}_{i} b_{i}+\mathbf{C} \boldsymbol{\beta}+\sum_{j} \mathbf{W}_{j} \mathbf{u}_{j}+\mathbf{e} \text { with } \mathbf{V}=\sum_{j} \mathbf{W}_{j} \mathbf{W}_{j}^{\prime} \sigma_{u_{j}}^{2}+\mathbf{I} \sigma_{e}^{2}
$$

where $\mathbf{W}_{j}$ is an $n \times m_{j}$ matrix of standardized DNAm measures of the probes in the $j$ th group with $m_{j}$ being the number of probes in the group (excluding probes within $50 \mathrm{~Kb}$ of the target probe). In practice, the probes are split into two groups by association $p$ values from a linear regression model (i.e., $\mathbf{y}=\mathbf{w}_{i} b_{i}+\mathbf{C} \boldsymbol{\beta}+\mathbf{e}$ ) at a methylome-wide significant threshold (all the methylome-wide significant probes in the first group and the other probes in the second group). The GLS method described in model [3] can be used to estimate $b_{i}$ and its SE for hypothesis testing. Like the exact MOA method, MOMENT is also computationally intensive when applied in a methylome-wide analysis. We can use a similar approximation approach as described above (i.e., using the variance components estimated under the null to compute $\hat{b}_{i}$ and SE) to reduce the computing cost. The variance components are re-estimated when one or more probes are excluded from the first group in case that the proportion of phenotypic variance captured by some of the probes in the first group are large. 


\section{Simulation analysis}

To quantify the false positive rate (or family-wise error rate) and statistical power of MOMENT (implemented in OSCA), we performed simulations based on DNAm and CTC [44] measures on samples from the Lothian Birth Cohorts (LBC) in three scenarios (Additional file 1: Note S1). We simulated a phenotype (1) with effects from a set of "causal probes" (randomly selected from all probes on the odd chromosomes) but no direct effects from the CTCs, (2) with small to large effects from CTCs but no effects from the probes, and (3) with effects from both the causal probes and CTCs (Additional file 1: Note S1). Note that we only sampled the causal probes from the odd chromosomes in scenarios 1 and 3, leaving the probes on the even chromosomes to quantify false positive rate under the null, and that the DNAm measures were adjusted for age, sex, experimental batches, and smoking status. Results from our models were compared to 6 different methods including (1) Unadj: linear regression without adjustment; (2) CTCadj: linear regression with CTCs fitted as covariates; (3) SVA: linear regression with the SVA surrogate variables fitted as covariates [22]; (4) LFMM2-ridge: a latent factor mixed model (LFMM) using ridge algorithm for confounder estimation [32]; (5) LFMM2-lasso: an LFMM using lasso algorithm for confounder estimation [32]; (6) ReFACTor: linear regression with the first 5 sparse principal components (PCs) from ReFACTor fitted as covariates [31]; (7) 5PCs: linear regression with the first $5 \mathrm{PCs}$, computed from a principal component analysis (PCA), fitted as covariates; and (8) FaST-LMM-EWASher: a set of selected probes fitted as random effect in an MLM [30]. For completeness of the analysis, we also included MOA (implemented in OSCA) in the comparison. We validated using a subset of data generated from simulation scenario 1 that the test statistics from the approximate MOA/MOMENT approach were extremely highly correlated with those from the corresponding exact approach (Pearson correlation > 0.999 for causal probes and $>0.998$ for null probes; Additional file 1: Figure S1). Hence, for the ease of computation, we used the approximate MOA/MOMENT approach in all the subsequent analyses.

In simulation scenario 1 , although there were no direct effects of the CTCs on the phenotype, the test statistics from Unadj at the null probes were inflated (Fig. 1a and Additional file 1: Table S1) because the null and causal probes-albeit on different sets of chromosomes-are correlated through their correlations with systematic biases such as CTCs. The mean genomic inflation factor $(\lambda)$ [45] of the null probes (on the even chromosomes) from 100 simulation replicates was 7.67 for Unadj (Additional file 1: Table S1), where $\lambda$ is defined as the median of $\chi^{2}$ test statistics of the null probes divided by its expected value. CTCadj reduced but not completely removed the inflation in test statistics of the null probes (Fig. 1a and Additional file 1: Table S1), suggesting that the inflation was caused by correlations between the causal and null probes because of the confounding effects of both CTCs and other unobserved confounders. While all the other methods were much less inflated compared to Unadj, MOMENT and MOA showed the least inflation with a mean $\lambda$ value close to 1 . It is slightly surprising to observe that the family-wise error rates (FWERs) of all the methods except MOA and MOMENT were highly inflated (FWERs >0.6) (Additional file 1: Figure S2a and Additional file 1: Table S1) despite the relatively small genomic inflation at the null probes for most of the methods (Fig. 1a). Here, FWER is defined as the proportion of simulation replicates with at least one null probe at MWAS $p$ value $<0.05 / m$ with $m$ being the number of null probes, which can be interpreted as the probability of observing one or more false positives at a methylome-wide significance level in a single experiment. There was no inflation in FWER for MOMENT, and a marginal inflation for MOA (Additional file 1: Figure S2a and Additional file 1: Table S1), showing the effectiveness of using all (distal) probes to account for the probe correlations. We also quantified the FPR, defined as the proportion of null probes with $p$ values $<0.05$ in each simulation replicate. The differences in FPR among the methods showed a similar pattern to the differences in genomic inflation factor (Additional file 1: Figure S2b and Additional file 1: Table S1). We then compared power among the methods. Since the test statistics of many approaches were highly inflated, it is not very meaningful to compare power without accounting for the inflation. We therefore used the area under the ROC curve (AUC) to compare power of the methods given the same level of FPR. Apart from Unadj and CTC, the AUCs of all the methods were on similar levels (Fig. 1b). The conclusions held in additional simulations varying the number of causal probes and the proportion of phenotypic variance captured by the causal probes (Additional file 1: Figure S3 and S4) despite that the inflation in FWER for the existing methods appeared to increase with the increase of the proportion of variance captured per causal probe. Additionally, we applied BACON, a summary-data-based method that seeks to remove genomic inflation taking the true positives into consideration, to the test statistics of all probes produced by the methods tested above. We showed that the inflation in test statistics of the null probes for Unadj was substantially reduced but not completely removed by the BACON adjustment and that the test statistics from MOA and MOMENT remained almost unchanged after the BACON adjustment (Additional file 1: Figure S5).

In simulation scenario 2 where there is no direct probetrait association, all the probes are null and their $\chi^{2}$ test statistics are expected to follow a $\chi^{2}$ distribution with 1 degree 


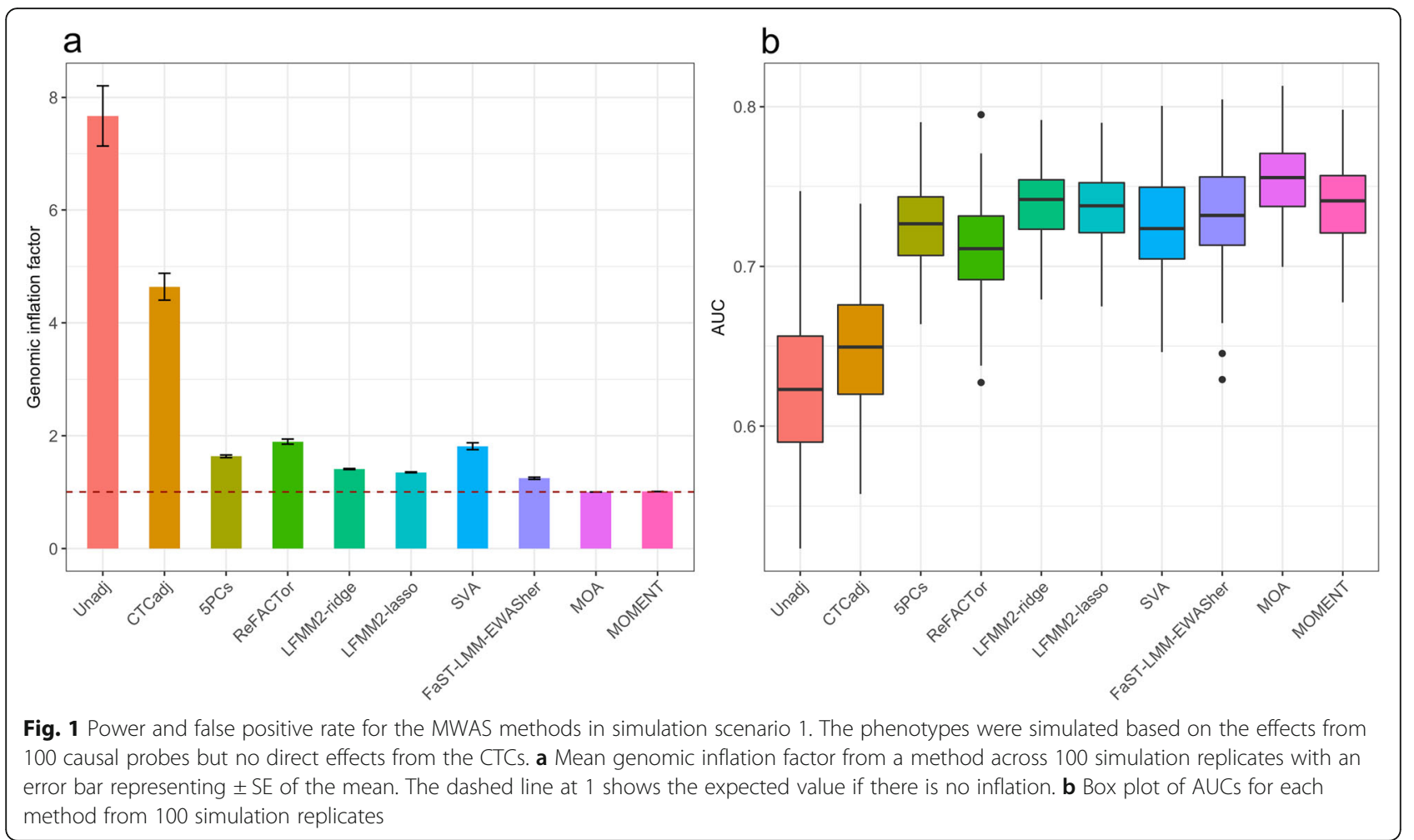

of freedom if the effects of CTCs have been well accounted for. The results showed that the $\lambda$ value was close to 1 for all the methods except Unadj and FaST-LMM-EWASher (Fig. 2a). It seems that, for some of the methods (e.g., 5PCs and ReFACTor), the $\lambda$ value slightly increased with the increase of the proportion of variance explained by the CTCs $\left(R_{\mathrm{CTCS}}^{2}\right)$ (Fig. 2a). The FPRs of the methods were highly consistent with the genomic inflation factors (Additional file 1: Figure S6). Nevertheless, a non-inflated median test statistic does not necessarily mean that the FWER has been well controlled for. In fact, most methods showed inflated FWER in this simulation scenario, and the FWERs of all the methods increased with increasing $R_{\mathrm{CTCs}}^{2}$ (Fig. 2b). The FWERs of 5PCs, ReFACTor, LFMM2-ridge, and LFMM2lasso were close to the expected value (i.e., 0.05) when $R_{\mathrm{CTCs}}^{2}=0.005$ and increased to a level between 0.15 and 0.2 when $R_{\mathrm{CTCs}}^{2}=0.05$ (Fig. 2b). The relationship between FWER and $R_{\text {CTCs }}^{2}$ was relatively flat for SVA with its FWER varying from 0.05 to 0.1 when $R_{\mathrm{CTCs}}^{2}$ increased from 0.005 to 0.05. Although FaST-LMM-EWASher showed the most deflated test statistics among all the methods (Fig. 2a), its FWER was substantially higher than all the other methods except Unadj (Fig. 2b), likely due to its feature selection strategy (Additional file 1: Note S2). MOA and MOMENT performed similarly in this simulation scenario and showed the lowest inflation in FWER among all the methods with their FWER being lower than 0.05 when $R_{\text {CTCs }}^{2}=0.005$ and increased to about 0.1 when $R_{\text {CTCs }}^{2}=0.05$ (Fig. 2b). In addition, we performed a linear regression analysis with the known CTCs fitted as covariates; as expected, the FWER was close to 0.05 irrespective of the level of $R_{\mathrm{CTCs}}^{2}$ (see below for the analysis with predicted CTCs).

We also compared the methods under the circumstance (simulation scenario 3 ) where there were associations between the phenotype and CTCs $\left(R_{\mathrm{CTCs}}^{2}=0.05\right)$ and the null probes were correlated with distal causal probes because both of them were correlated with CTCs (Additional file 1: Note S1). The results were similar to those above (Fig. 1 and Additional file 1: Figure S2). That is, the FWER of MOMENT was close to the expected value, demonstrating the reliability and robustness of the method. The FWER of MOA is slightly higher than that of MOMENT but much lower than those of the other methods which showed strong inflation in FWER and/or FPR due to the correlations between causal and null probes (Additional file 1: Figure S7a, S7c, and S7d, and Additional file 1: Table S2). All the methods showed similar levels of AUC except for Unadj and CTCadj (Additional file 1: Figure S7b). The conclusions held with different sample sizes (Additional file 1: Figure S8 and S9) or different numbers of causal probes with smaller or larger variance explained per causal probe (Additional file 1: Figure S10 and S11). The conclusions also held if we simulated confounding 

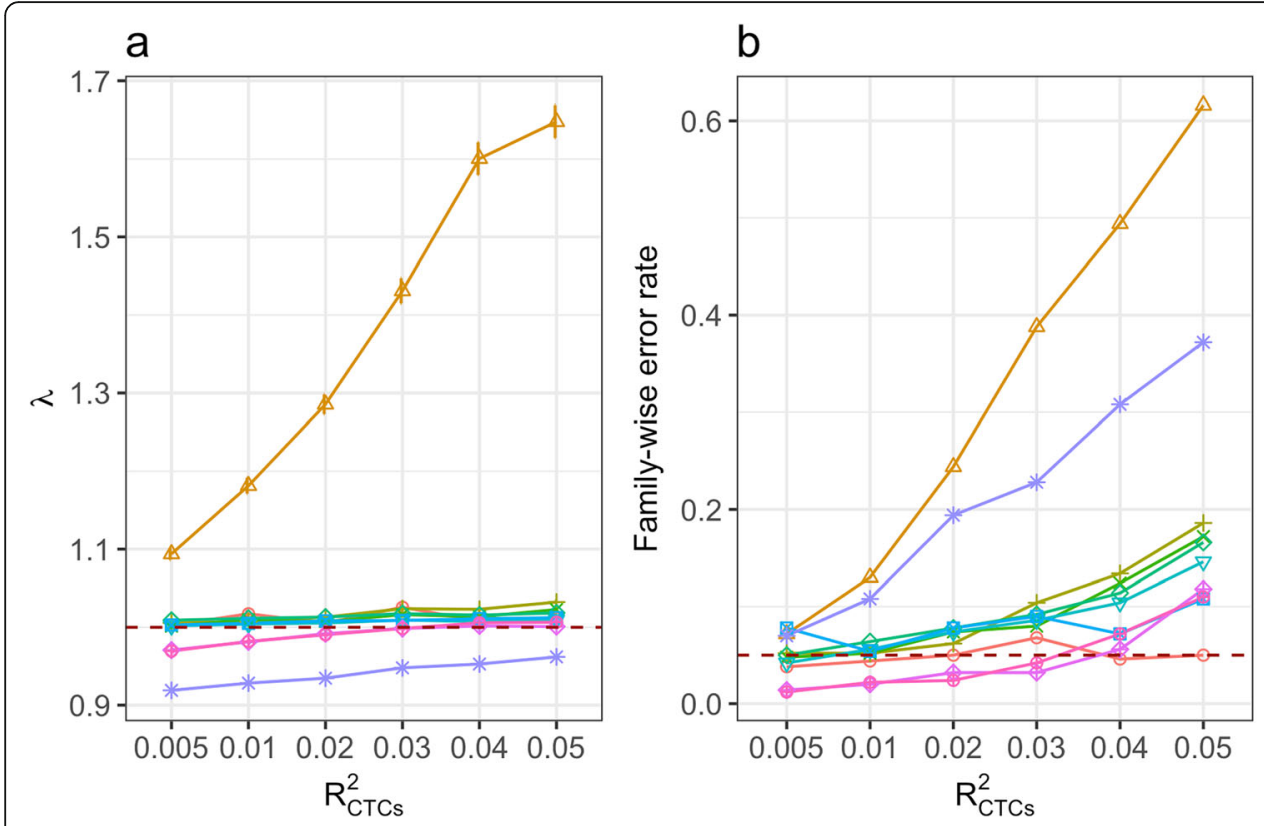

Method

$\rightarrow$ CTCadj

$\triangle$ Unadj

$+5 \mathrm{PCs}$

$*$ ReFACTor

$\diamond$ LFMM2-ridge

$\nabla$ LFMM2-lasso

- - SVA

* FaST-LMM-EWASher

$\rightarrow$ MOA

$\rightarrow$ MOMENT

Fig. 2 Genomic inflation factor and family-wise error rate for the MWAS methods in simulation scenario 2 (effects from CTCs but no causal probes). Shown on the horizontal axis are the $R_{\text {CTCS }}^{2}$ values used to simulate the phenotype. a Each dot represents the mean $\lambda$ value from 1000 simulation replicates given a specified $R_{C T C S}^{2}$ value for a method with an error bar representing \pm SE of the mean. $\mathbf{b}$ Each dot represents the family-wise error rate, calculated as the proportion of simulation replicates with one or more null probes detected at a methylome-wide significance level

effects on experimental batches in lieu of CTCs (Additional file 1: Figure S12 and S13). We further demonstrated that the result from MOA/MOMENT analysis of the whole sample was consistent with that from a metaanalysis of summary statistics from MOA/MOMENT analyses in two halves of the sample (Additional file 1: Figure S14) and that the methods were applicable to case-control phenotypes (Additional file 1: Figure S15 and S16).

To explore the applicability of the proposed methods to other types of omic data, we tested the methods by simulation based on a real gene expression data set (19, 648 gene expression probes on 1219 Mexican American individuals) from the San Antonio Family Heart Study (SAFHS) [46-48] ("Methods" section) under simulation scenario 1 (i.e., quantitative phenotypes simulated based on the expression levels of 100 randomly selected causal probes; Additional file 1: Note S1). The result showed that both MOMENT and MOA performed similarly (in comparison to the other methods) as in the simulations based on DNAm data (Additional file 1: Figure S17).

We further compared the computational complexity among the MWAS methods tested in this study and quantified their runtime and memory usage of the methods using simulated and real phenotypes in the LBC (Additional file 1: Table S3). We found that MOA and MOMENT showed the lowest memory usage among all the methods. The approximate MOA approach was the second fastest approach (only slightly slower than LFMM2-ridge), and the approximate MOMENT approach was slower than LFMM2-ridge, approximate MOA, and ReFACTor but much faster than SVA, LFMM2-lasso, and EWASher.

\section{An application of MOMENT to real data}

We applied MOMENT and the other methods to four real quantitative traits in the $\mathrm{LBC}$ cohorts. These traits, including BMI, height, lung function (measured in the highest score of forced expiratory volume in $1 \mathrm{~s}$ ), and walking speed (measured in the time taken to walk $6 \mathrm{~m}$ ), were standardized and corrected for age in each gender group within each sub-cohort (LBC1936 or LBC1921) ("Methods" section). The standardized phenotypes were further processed by a rank-based inverse-normal transformation. The DNAm probes were adjusted for age, sex, and experimental batches. We did not adjust the probes for CTCs or smoking status for the purpose of testing methods (see below).

Consistent with the results from simulations, the test statistics from MOA and MOMENT were not inflated whereas all the other methods showed modest inflation for all the traits (Fig. 3, Table 1, and Additional file 1: Figure S18-S21). Three associations were identified by multiple methods, including one for BMI (cg11202345, detected by all methods), in line with a previous study [49], and two for lung function ( $\operatorname{cg} 05575921$ and 

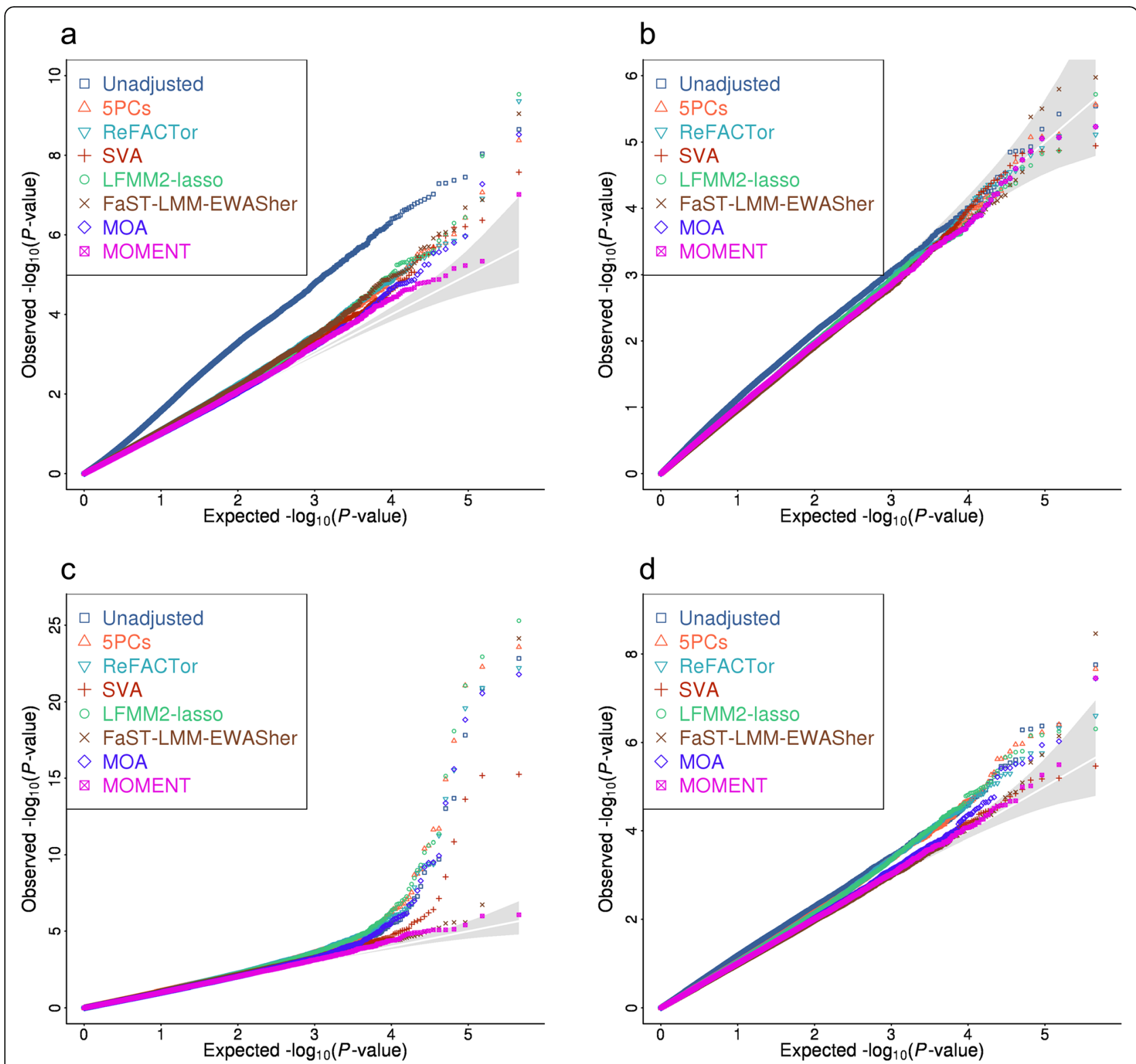

Fig. $3 \mathrm{QQ}$ plot of $p$ values from MWAS analysis for 4 quantitative traits in the LBC data. The DNAm measures were adjusted for age, sex, and batches. The phenotypes were stratified into groups by sex and cohort and were adjusted for age and standardized to $z$-scores by rank-based inverse normal transformation in each group. The phenotypes are $\mathbf{a}$ BMI, b height, $\mathbf{c}$ lung function, and $\mathbf{d}$ walking speed

cg05951221, detected by all methods except MOMENT) (Additional file 1: Table S4, Additional file 1: Figure S18 and S20). It should be noted that cg05575921 was reported to be associated with smoking in a previous study [50], indicating that the association between cg05575921 and lung function might be confounded by smoking status. Moreover, MOA, LFMM2-ridge, LFMM2-lasso, and ReFACTor consistently identified 12 additional probes associated with lung function but most of the probes have been linked to smoking in a previous study [51]. Almost all the associations were not significant when smoking status was fitted as a covariate in the models
(6.5\% of variance in lung function associated with smoking status, Additional file 1: Table S5 and Additional file 1: Figure S22), suggesting that most (if not all) of the probe associations with lung function identified by MOA, LFMM2-ridge, LFMM2-lasso, and ReFACTor were owing to the confounding of smoking. None of the smokingassociated probes were methylome-wide significant for lung function in the analysis using MOMENT (Additional file 1: Figure S20), and the result remained the same when smoking status was fitted as a covariate in MOMENT (Additional file 1: Figure S22), again demonstrating the capability of MOMENT in correcting for 
Table 1 Genomic inflation factors reported by different MWAS methods for the 4 traits in the Lothian Birth Cohorts

\begin{tabular}{lllll}
\hline & BMI & Height & Lung function & Walking speed \\
\hline Unadjusted & 1.68 & 1.30 & 0.98 & 1.28 \\
5PCS & 1.11 & 0.96 & 1.06 & 1.04 \\
SVA & 1.04 & 0.95 & 1.06 & 1.01 \\
LFMM2-ridge & 1.09 & 1.00 & 1.10 & 1.04 \\
LFMM2-lasso & 1.08 & 0.99 & 1.09 & 1.03 \\
ReFACTor & 1.13 & 0.97 & 1.09 & 1.02 \\
EWASher & 1.11 & 0.96 & 1.09 & 0.96 \\
MOA & 0.99 & 1.02 & 0.97 & 0.99 \\
MOMENT & 1.00 & 1.02 & 0.98 & 1.00 \\
\hline
\end{tabular}

unobserved confounding factors. This is further supported by the finding from simulations that the effects of null probes estimated from MOMENT were much less correlated with the phenotype compared to those estimated from MOA (Additional file 1: Figure S23).

It has been shown in previous GWASs that MLMbased association analysis methods developed for quantitative traits are applicable to case-control data [37-39, 52]. We have shown by simulation that both MOMENT and MOA are applicable to case-control phenotypes regardless whether cases are oversampled (Additional file 1: Figure S15 and S16). To demonstrate the applicability of the proposed methods to discrete phenotypes, we analyzed smoking status (coded as 0,1 , or 2 for nonsmoker, former smoker, or current smoker) in the LBC by MOA and MOMENT in comparison with existing methods. All the methods detected a large number (at least 112) of probes at a methylome-wide significance level $(p<2.19 \mathrm{e}-7)$ except for MOMENT and EWASher which only identified 4 and 2 probes, respectively, at the methylome-wide significance level (Additional file 1: Figure S24). To validate the association signals other than those identified by MOMENT, we fitted the $4 \mathrm{MO}$ MENT probes as fixed covariates in MOA. None of the additional associations remained methylome-wide significant conditioning on the 4 MOMENT probes (Additional file 1: Figure S25), suggesting that those additional associations detected by MOA (and other methods) were driven by their correlations with the 4 MOMENT signals. MOA failed in this scenario likely because the associations of the 4 MOMENT signals were too strong to be fitted in a single normal distribution with the other probes. This conclusion is further supported by the result that the accuracy of predicting/classifying smoking status in a cross-validation setting using a large number of probes detected by linear regression or MOA was even lower than that using a small number of probes detected by MOMENT (Additional file 1: Table S6). In addition, we recoded the smoking status data to a binary phenotype ( 0 for non-smoker and 1 for former or current smoker) and applied all the methods to the recoded binary phenotype; the conclusions were similar as above but it seemed that the analyses with the binary phenotype were less powerful than those with the categorical phenotype above (Additional file 1: Figure S26). All these results show the applicability of MOMENT to discrete traits and again demonstrate the robustness and reliability of MOMENT in controlling for false positive associations.

\section{Estimating variance in a phenotype captured by all probes by OREML}

We have demonstrated the performance of the omic-databased association analysis methods in OSCA by simulation and real data analysis. We then turned to evaluate the performance of OREML in estimating the proportion of variance in a complex trait captured by all probes $\left(\rho^{2}\right)$ by simulation in two scenarios (Additional file 1: Note S1). The results showed that under either scenario, OREML reported an unbiased estimate of $\rho^{2}$ (Additional file 1: Table S7). Here, the unbiasedness is defined as that the mean $\rho^{2}$ estimate from 500 independent simulations is not significantly different from the $\rho^{2}$ parameter used for simulation. There are two methods implemented in OSCA to compute the ORM ("Methods" section). Our simulation results showed that the estimates of $\rho^{2}$ based on the two methods were similar (Additional file 1: Table S7).

We also attempted to partition and estimate the proportions of phenotypic variation captured by all SNPs (i.e., $h_{\mathrm{SNP}}^{2}$ ) and all the DNAm probes respectively when fitted jointly in a model. We first investigated the correlation between genomic relationship matrix (GRM) and methylomic relationship matrix (MRM) in the LBC dataset. We found that the off-diagonal elements of the GRM were almost independent of those of the MRM ( $r$ $=0.0045$; Additional file 1: Figure S27). From an OREML analysis that fits both the GRM and MRM, we estimated that all the DNAm probes captured 6.5\% (SE = 0.038) of the variance for BMI but the estimate for height was nearly zero $\left(\hat{\rho}^{2}=-0.005\right.$ and $\left.\mathrm{SE}=0.0086\right)$ (Additional file 1: Table S8). These results are in line with the finding from a previous study that the accuracy of genetic risk prediction can be improved by incorporating DNAm data for BMI but not height [14].

\section{Discussion}

In this study, we developed a versatile software toolOSCA - to manage omic data generated from highthroughput experiments in large cohorts and to facilitate the analyses of complex traits using omic data (Additional file 1: Note S4). The primary applications of OSCA are to identify omic measures associated with a 
complex trait accounting for unobserved confounding factors (MOMENT) and to estimate the proportion of phenotypic variation captured by all measures of one or multiple omic profiles (OREML). A by-product of the OREML application is to estimate the joint effects of all measures of one or multiple omic profiles (i.e., OBLUP analysis) to predict the phenotype in a new sample. This has been shown to be a powerful and robust approach in age prediction using gene expression or DNAm data [53, 54]. We have also provided computationally efficient implementations in OSCA to manage large-scale omic data and to perform omic-data-based quantitative trait locus (xQTL) analysis and meta-analysis of xQTL summary data. OSCA is an ongoing software development project so that any further methods or functions related to omic-data-based analysis can be included in the software package in the future.

We showed, by simulation, a surprisingly high error rate for all the existing MWAS/EWAS methods, mainly owing to the correlations between distal probes induced by CTCs (and/or other systematic confounders) in DNAm data (Fig. 1). These correlations are widespread at a large number of probes across the methylome (as demonstrated by the proportion of null probes with $P_{\text {MWAS }}<0.05$ in simulation scenario 1; Additional file 1: Figure S28) and thus are not adequately accounted for by a fixed number of principal features computed from the data (e.g., 5PCs, ReFACTor, LFMM2, and SVA) nor a set of selected probes (e.g., FaSTLMM-EWASher). This conclusion is likely to be applicable to other types of omic data if the measures in distal genomic regions are correlated due to unmeasured confounding factors such as systematic experimental biases or unwanted biological variation, as suggested by our simulations with gene expression data (Additional file 1: Figure S17). This confounding effect can be corrected for by fitting the target probe as a fixed effect and all the other (distal) probes as random effects (i.e., the MOA or MOMENT method). In addition, we tested the robustness of MOMENT to the change of window size used to exclude probes in close physical proximity to the target probe in either direction. We varied the window size from $100 \mathrm{kbp}$ to $250 \mathrm{bp}$ in the MOMENT analysis of data generated from simulation scenario 1 (Additional file 1: Figure S29). We found that the results remained almost unchanged when the window sizes decreased from 100 to $25 \mathrm{kbp}$ whereas there were a substantial number of probes showing deflated test statistics when the window size decreased to $500 \mathrm{bp}$ or 250 bp (Additional file 1: Figure S29). These results justify the use of $50 \mathrm{kbp}$ as the default window size for MOMENT when applied to DNAm data. We also quantified the decay of correlation between a pair of gene expression probes as a function of their physical distance (Additional file 1: Figure S30), which suggests that $100 \mathrm{kbp}$ is an appropriate MOMENT window size for gene expression data although the results remained almost unchanged when the window size was varied from $50 \mathrm{kbp}$ to $1 \mathrm{Mbp}$ in the simulated data (Additional file 1: Figure S31).

Our simulation also showed that, if CTCs or batches explain a large proportion of variation in the phenotype, the FWERs of all the methods tended to be inflated (Additional file 1: Figure S32 and S33) despite that the genomic inflation factor is close to unity for most methods (Fig. 2). We re-ran the simulation under a more extreme setting with $R_{\text {CTCs }}^{2}$ varying from 10 to $70 \%$. In this case, the genomic inflation factors of the fixed-effect models (i.e., SVA, ReFACTor, LFMM2, and 5PCs) and the FWERs of all the methods increased as $R_{\text {CTCs }}^{2}$ increased (to a lesser extent for FaST-LMM-EWASher), suggesting that there were a set of probes strongly associated with CTCs (Additional file 1: Figure S34). Note that even in this extreme case, MOMENT showed the lowest FWERs on average among all the methods. It is also of note that the FWERs of FaSTLMM-EWASher were relatively low in this scenario (Additional file 1: Figure S32), opposite to its performance when $R_{\text {CTCs }}^{2}$ was low (Fig. 2), possibly due to its variable selection strategy (Additional file 1: Note S2). The inflation in FWER was only slightly alleviated by fitting the predicted CTCs as covariates (Additional file 1: Figures S35 and S36). The results also suggest that it may be worth fitting measured CTCs as fixed-effect covariates in MLM-based association analyses such as MOA and MOMENT in practice although this approach is likely to be conservative as indicated by the deflated $\lambda$ and FWER (Additional file 1: Figure S37). These conclusions are likely to be applicable to other confounding factors such as smoking status, as demonstrated in the analysis of lung function data in the LBC (Additional file 1: Figure S22). Our results also caution the interpretation of associations identified from MWAS for traits that are highly correlated with CTCs and/or other biological confounders. In addition, although our simulation shows that both MOMENT and MOA are applicable to case-control phenotypes (Additional file 1: Figures S15 and S16), direct application of linear model approaches to $0 / 1$ traits is not ideal. If the underlying model is causal (i.e., omic measures have causal effects on the trait), a more appropriate analysis is to use a link function (e.g., a probit or logit model) that connects the $0 / 1$ phenotype to a latent continuous trait, as in the methods recently developed for the analysis of casecontrol data in GWAS [55-58]. Since OSCA is an ongoing software development project, the non-linear link functions can be incorporated in the MOMENT/MOA framework in the future.

In conclusion, we showed by simulation the inflation in test statistics of the existing MWAS methods because of the ubiquitous correlations between distal probes caused by confounding factors, and developed two new MWAS methods (MOA and MOMENT) to correct for the inflation. We demonstrated the reliability and robustness of 
MOMENT by simulations in a number of scenarios and real data analyses. We recommend the use of MOMENT in practice because of its robustness in the presence of unobserved confounders despite that it is slightly less powerful than MOA. We implemented both MOA and MOMENT in a computationally efficient and easy-to-use software tool OSCA together with many other functions for omic-databased analyses (Additional file 1: Figure S38).

\section{Methods}

\section{Omic-data-based relationship matrix (ORM)}

We have described in Eqs. $(1,2)$ the OREML model to estimate the proportion of variance in a phenotype captured by the DNAm probes all together. In Eq. (1), i.e., $\mathbf{y}=\mathbf{C} \boldsymbol{\beta}+$ $\mathbf{W u}+\mathbf{e}$, we define $\mathbf{W}$ as a matrix of standardized DNAm measures of all probes, and in Eq. (2), we define the ORM as $\mathbf{A}=\mathbf{W} \mathbf{W}^{\prime} / m$. Therefore, the omic relationship between individual $j$ and $k$ (the $j k$ th element of $\mathbf{A}$ ) can be computed as $A_{j k}=\frac{1}{m} \sum_{i}\left(x_{i j}-\mu_{i}\right)\left(x_{i k}-\mu_{i}\right) / \sigma_{i}^{2}$, where $x_{i j}$ is the unstandardized DNAm level of probe $i$ in individual $j$, $\mu_{i}$ and $\sigma_{i}^{2}$ are the mean and variance of the $i$ th probe over all the individuals respectively, and $m$ is the number of probes. This model implicitly assumes that the probes of smaller variance in DNAm level (unstandardized) tend to have larger effects on the phenotype (strictly speaking, stronger associations with the phenotype) and that there is no relationship between the proportion of trait variance captured by a probe and the variance of the probe. We also provide in OSCA an alternative method to compute the ORM, i.e., $A_{j k}=\sum_{i}\left(x_{i j}-\mu_{i}\right)\left(x_{i k}-\mu_{i}\right) / \sum_{i} \sigma_{i}^{2}$. If we use this definition of ORM in the OREML analysis, we implicitly assume that there is no relationship between the probe effect on the trait and the variance of the probe but the proportion of trait variance associated with a probe increases as the variance of the probe increases. We showed by simulation and real data analysis that the difference between OREML results using the two methods was very small (Additional file 1: Tables S7 and S8).

\section{OREML: estimating the proportion of trait variance captured by all DNAm probes}

We have shown in Eqs. $(1,2)$ an OREML model with one random-effect component to estimate the proportion of trait variance captured by all DNAm probes. The model is flexible, which can be extended to partition the trait variance into components associated with different sets of probes (e.g., a model with 22 components with all the probes on each chromosome as a component). A flexible OREML model can be written as.

$$
\begin{aligned}
& \quad \mathbf{y}=\mathbf{C} \boldsymbol{\beta}+\sum_{i} \mathbf{W}_{i} \mathbf{u}_{i}+\mathbf{e} \text { with } \quad \operatorname{var}(\mathbf{y})=\mathbf{V}=\sum_{i} \mathbf{W}_{i} \mathbf{W}_{i}{ }^{\prime} \sigma_{u_{i}}^{2} \\
& +\mathbf{I} \sigma_{e}^{2}=\sum_{i} \mathbf{A}_{i} \sigma_{o_{i}}^{2}+\mathbf{I} \sigma_{e}^{2} .
\end{aligned}
$$

where the definitions of all the parameters and variables are similar to those in Eqs. $(1,2)$. The variance components can be estimated by REML [33], and the proportion of the trait variance captured by the $i$ th component can be computed as $\rho_{i}^{2}=\sigma_{o_{i}}^{2} /\left(\sum_{i} \sigma_{o_{i}}^{2}+\sigma_{e}^{2}\right)$.

The multi-component OREML model can be applied to partition the trait variance into components associated with multiple omic profiles. For example, if SNP genotype, DNAm, and gene expression data are available for all the individuals in a cohort, a multi-component OREML model can be used to estimate the proportion of trait variance captured by all SNPs (i.e., the SNP-based heritability), the expression levels of all genes, and the DNAm levels at all the CpG sites. The model can be written as $\mathbf{y}=\mathbf{C} \boldsymbol{\beta}+\mathbf{W}_{g} \mathbf{u}_{g}+\mathbf{W}_{t} \mathbf{u}_{t}+\mathbf{W}_{m} \mathbf{u}_{m}$ $+\mathbf{e}$ with $\operatorname{var}(\mathbf{y})=\mathbf{A}_{g} \sigma_{g}^{2}+\mathbf{A}_{t} \sigma_{t}^{2}+\mathbf{A}_{m} \sigma_{m}^{2}+\mathbf{I} \sigma_{e}^{2}$

where $\mathbf{W}_{g}, \mathbf{W}_{t}$, and $\mathbf{W}_{m}$ are the matrices of standardized SNP genotypes, gene expression measures, and DNAm levels, respectively, with the corresponding effects $\mathbf{u}_{g}$, $\mathbf{u}_{t}$, and $\mathbf{u}_{m} ; \mathbf{A}_{g}=\mathbf{W}_{g} \mathbf{W}_{g}^{\prime} / m_{g}$ is the genomic relationship matrix (GRM) with $m_{g}$ being the number of SNPs, $\mathbf{A}_{t}$ $=\mathbf{W}_{t} \mathbf{W}_{t}^{\prime} / m_{t}$ is the transcriptomic relationship matrix (TRM) with $m_{t}$ being the number of transcripts, and $\mathbf{A}_{m}$ $=\mathbf{W}_{m} \mathbf{W}_{m}^{\prime} / m_{m}$ is the methylomic relationship matrix (MRM) with $m_{m}$ being the number of DNAm probes. Note that the model can be reduced by dropping any of the variance components or expanded by including other types of omic profiles (e.g., protein abundance).

\section{Dataset}

The LBC cohorts $[59,60]$ consisted of individuals born in 1921 (LBC1921) and 1936 (LBC1936), mostly living in Edinburgh city and the surrounding Lothian region of Scotland. Blood samples were collected with informed consent. The LBC individuals underwent several waves of SNP genotyping and DNAm measures. DNAm levels at 485,512 CpG sites across the genome were measured on 3191 whole blood samples from 3 waves using the Illumina HumanMethylation450 BeadChip. Duplicates or samples with an excessive proportion of low confidence calls across all probes $(>5 \%)$ were removed. Probes with an excessive proportion of low confidence calls across all individuals $(>5 \%)$ or probes located in sex chromosomes were excluded. In addition, probes encompassing SNPs annotated in dbSNP131 using hg19 coordinates or identified as potentially cross-hybridized methylation probes by a previous study [61] were also excluded. After these QC steps, 3018 samples and 307,360 probes remained (Additional file 1: Note S3). We included in the analysis only the first wave (wave1) of the LBC data consisting of 436 individuals from LBC1921 (average age of 79 years) and 906 individuals from LBC1936 (average age of 70 years) (Additional file 1: Table S9). We removed probes with almost invariable beta values across individuals (standard 
deviation < 0.02) and retained 1342 individuals and 228, 694 probes for analysis.

There were a number of covariates available in the LBC data including age, sex, batches of the experiment (i.e., plate and position of the sample on a chip), and CTCs. The blood cell counts for different cell types, including basophils, eosinophils, monocytes, lymphocytes, and neutrophils, were quantified using an LH50 Beckman Coulter instrument on the same day of blood collection. In addition to the covariates, there are a number of traits measured on the LBC individuals including height (measured without shoes), body mass index (BMI), lung function (measured in the highest score of forced expiratory volume in $1 \mathrm{~s}$ ), walking speed (measured in the time taken to walk $6 \mathrm{~m}$ ), and smoking status (never smoked, ex-smoker, or current smoker) [62, 63]. The numbers of missing measurements are noted in Additional file 1: Table S10. For each trait, we adjusted the phenotype for age in each gender group of each cohort (LBC1921 or LBC1936) and standardized the residuals by rank-based inverse normal transformation, which removed the age effect and potential difference in mean and variance between two gender groups or cohorts.

The LBC wave1 individuals were also genotyped by Illumina 610-Quadv1 BeadChip. The QC process of the SNP genotype data has been detailed elsewhere [14]. After excluding SNPs from sex chromosomes and SNPs with low allelic frequency (MAF $<0.01$ ), we retained 523 , 062 genotyped SNPs for analysis.

We also used a set of gene expression data available at EMBL-EBI (URLs) from the San Antonio Family Heart Study (SAFHS). Sample recruitment, data generation, and quality controls of the SAFHS data have been detailed elsewhere [46-48]. We used the processed and standardized gene expression data of 19,648 autosomal probes on 1240 non-diseased Mexican American participants. Age, sex, and smoking status were available in the data. We removed 21 samples with unknown smoking status and retained 1219 individuals for analysis.

\section{URLS}

OSCA, http://cnsgenomics.com/software/osca

ReFACTor, https://www.cs.tau.ac.il/ heran/cozygene/ software/refactor.html

EWASher, https://www.microsoft.com/en-us/research/ project/fast-lmm-software-papers/

SVA, https://bioconductor.org/packages/release/bioc/ html/sva.html

LFMM2, https://bcm-uga.github.io/lfmm/

The LBC data: https://www.ebi.ac.uk/ega/studies/ EGAS00001000910

The SAFHS data: https://www.ebi.ac.uk/arrayexpress/ experiments/E-TABM-305/

\section{Additional files}

Additional file 1: Figures S1-S38, Tables S1-S12, and Notes S1-S4. (PDF 17946 kb)

Additional file 2: Review history. (DOCX $21 \mathrm{~kb}$ )

Review history

The review history is available as Additional file 2.

Authors' contributions

JY conceived the study. JY and FZ designed the experiment. FZ developed the software tool and performed all the analyses under the guidance and/or assistance from JY, ZZ, WC, QZ, MFN, and TQ. JY, AFM, PMV, and NRW contributed funding and resources. IJD, PMV, and NRW contributed the DNA methylation data. FZ, JY, and WC wrote the manuscript with the participation of all authors. All authors read and approved the final manuscript.

\section{Funding}

This research was supported by the Australian Research Council (FT180100186), the Australian National Health and Medical Research Council (grants 1107258 , 1113400, 1083656, 1078037, and 1078901), and the Sylvia \& Charles Viertel Charitable Foundation. The Lothian Birth Cohorts (LBC) are supported by Age UK (Disconnected Mind program). Methylation typing was supported by Centre for Cognitive Ageing and Cognitive Epidemiology (Pilot Fund award), Age UK, The Wellcome Trust Institutional Strategic Support Fund, The University of Edinburgh, and The University of Queensland. The LBC resource is prepared in the Centre for Cognitive Ageing and Cognitive Epidemiology, which is supported by the Medical Research Council and Biotechnology and Biological Sciences Research Council (MR/K026992/1), and which supports I.J.D..

\section{Availability of data and materials}

This study makes use of DNA methylation data from the LBC available at EGA (accession: EGAS00001000910) [64] and gene expression data from the SADHS available at EMBL-EPI (accession: E-TABM-305, [65]). The source code of OSCA is available at a DOl-assigning repository Zenodo (https://doi.org/10. 5281/zenodo.2658802) [66] and at GitHub (https://github.com/jianyangqt/ osca) under the GNU General Public License v3.0.

\section{Ethics approval and consent to participate}

The use of human data in this study was approved by The University of Queensland Human Research Ethics Committee B (approval number: 2011001173).

Consent for publication

Not applicable.

\section{Competing interests}

The authors declare that they have no competing interests.

\section{Author details}

${ }^{1}$ Institute for Molecular Bioscience, The University of Queensland, Brisbane, Queensland 4072, Australia. ${ }^{2}$ University of Exeter Medical School, Devon EX2 5DW, UK. ${ }^{3}$ Centre for Cognitive Ageing and Cognitive Epidemiology, Department of Psychology, University of Edinburgh, 7 George Square, Edinburgh EH8 9JZ, UK. ${ }^{4}$ Queensland Brain Institute, The University of Queensland, Brisbane, Queensland 4072, Australia. ${ }^{5}$ Institute for Advanced Research, Wenzhou Medical University, Wenzhou 325027, Zhejiang, China.

Received: 16 January 2019 Accepted: 20 May 2019

Published online: 28 May 2019

References

1. Ritchie MD, Holzinger ER, Li R, Pendergrass SA, Kim D. Methods of integrating data to uncover genotype-phenotype interactions. Nat Rev Genet. 2015;16:85-97.

2. Hasin Y, Seldin M, Lusis A. Multi-omics approaches to disease. Genome Biol. 2017;18:83.

3. Wu Y, Zeng J, Zhang F, Zhu Z, Qi T, Zheng Z, Lloyd-Jones LR, Marioni RE, Martin NG, Montgomery GW, et al. Integrative analysis of omics summary 
data reveals putative mechanisms underlying complex traits. Nat Commun. 2018;9:918.

4. Consortium GT, Laboratory DA, Coordinating Center -Analysis Working G, Statistical Methods groups-Analysis Working G, Enhancing Gg, Fund NIHC, Nih/Nci, Nih/Nhgri, Nih/Nimh, Nih/Nida, et al. Genetic effects on gene expression across human tissues. Nature. 2017:550:204-13.

5. Lloyd-Jones LR, Holloway A, McRae A, Yang J, Small K, Zhao J, Zeng B, Bakshi A, Metspalu A, Dermitzakis M, et al. The genetic architecture of gene expression in peripheral blood. Am J Hum Genet. 2017;100:371.

6. Hannon E, Spiers H, Viana J, Pidsley R, Burrage J, Murphy TM, Troakes C, Turecki G, O'Donovan MC, Schalkwyk LC, et al. Methylation QTLs in the developing brain and their enrichment in schizophrenia risk loci. Nat Neurosci. 2016;19:48-54.

7. Jaffe AE, Gao Y, Deep-Soboslay A, Tao R, Hyde TM, Weinberger DR, Kleinman JE. Mapping DNA methylation across development, genotype and schizophrenia in the human frontal cortex. Nat Neurosci. 2016;19:40-7.

8. Grubert F, Zaugg JB, Kasowski M, Ursu O, Spacek DV, Martin AR, Greenside P, Srivas R, Phanstiel DH, Pekowska A, et al. Genetic control of chromatin states in humans involves local and distal chromosomal interactions. Cell. 2015;162:1051-65.

9. Chen L, Ge B, Casale FP, Vasquez L, Kwan T, Garrido-Martin D, Watt S, Yan Y, Kundu K, Ecker S, et al. Genetic drivers of epigenetic and transcriptional variation in human immune cells. Cell. 2016;167:1398-1414 e1324.

10. Battle A, Khan Z, Wang SH, Mitrano A, Ford MJ, Pritchard JK, Gilad Y. Genomic variation. Impact of regulatory variation from RNA to protein. Science. 2015;347:664-7.

11. Folkersen L, Fauman E, Sabater-Lleal M, Strawbridge RJ, Franberg M, Sennblad B, Baldassarre D, Veglia F, Humphries SE, Rauramaa R, et al. Mapping of 79 loci for 83 plasma protein biomarkers in cardiovascular disease. PLoS Genet. 2017;13:e1006706.

12. Wahl S, Drong A, Lehne B, Loh M, Scott WR, Kunze S, Tsai PC, Ried JS, Zhang W, Yang Y, et al. Epigenome-wide association study of body mass index, and the adverse outcomes of adiposity. Nature. 2017;541:81-6.

13. Gusev A, Mancuso N, Won H, Kousi M, Finucane HK, Reshef Y, Song L, Safi A, Schizophrenia Working Group of the Psychiatric Genomics C, McCarroll S, et al. Transcriptome-wide association study of schizophrenia and chromatin activity yields mechanistic disease insights. Nat Genet. 2018;50:538-48.

14. Shah S, Bonder MJ, Marioni RE, Zhu Z, McRae AF, Zhernakova A, Harris SE, Liewald D, Henders AK, Mendelson MM, et al. Improving phenotypic prediction by combining genetic and epigenetic associations. Am J Hum Genet. 2015;97:75-85.

15. van Kessel KEM, van der Keur KA, Dyrskjot L, Algaba F, Welvaart NYC, Beukers W, Segersten U, Keck B, Maurer T, Simic T, et al. Molecular markers increase precision of the European Association of Urology non-muscle-invasive bladder cancer progression risk groups. Clin Cancer Res. 2018;24:1586-93.

16. Gamazon ER, Wheeler HE, Shah KP, Mozaffari SV, Aquino-Michaels K, Carroll RJ, Eyler AE, Denny JC, Consortium GT, Nicolae DL, et al. A gene-based association method for mapping traits using reference transcriptome data. Nat Genet. 2015;47:1091-8.

17. Gusev A, Ko A, Shi H, Bhatia G, Chung W, Penninx BW, Jansen R, de Geus EJ, Boomsma DI, Wright FA, et al. Integrative approaches for large-scale transcriptome-wide association studies. Nat Genet. 2016;48:245-52.

18. Zhu Z, Zhang F, Hu H, Bakshi A, Robinson MR, Powell JE, Montgomery GW, Goddard ME, Wray NR, Visscher PM, Yang J. Integration of summary data from GWAS and eQTL studies predicts complex trait gene targets. Nat Genet. 2016:48:481-7.

19. Liu Y, Aryee MJ, Padyukov L, Fallin MD, Hesselberg E, Runarsson A, Reinius L, Acevedo N, Taub M, Ronninger M, et al. Epigenome-wide association data implicate DNA methylation as an intermediary of genetic risk in rheumatoid arthritis. Nat Biotechnol. 2013;31:142-7.

20. Michels KB, Binder AM, Dedeurwaerder S, Epstein CB, Greally JM, Gut I, Houseman EA, Izzi B, Kelsey KT, Meissner A, et al. Recommendations for the design and analysis of epigenome-wide association studies. Nat Methods. 2013;10:949-55.

21. Jaffe AE, Irizarry RA. Accounting for cellular heterogeneity is critical in epigenome-wide association studies. Genome Biol. 2014;15(2):R31.

22. Leek JT, Storey JD. Capturing heterogeneity in gene expression studies by surrogate variable analysis. PLoS Genet. 2007;3:1724-35.

23. Teschendorff AE, Zheng SC. Cell-type deconvolution in epigenome-wide association studies: a review and recommendations. Epigenomics. 2017; 9:757-68.
24. Teschendorff AE, Relton CL. Statistical and integrative system-level analysis of DNA methylation data. Nat Rev Genet. 2018;19:129-47.

25. Rakyan VK, Beyan H, Down TA, Hawa Ml, Maslau S, Aden D, Daunay A, Busato F, Mein CA, Manfras B, et al. Identification of type 1 diabetesassociated DNA methylation variable positions that precede disease diagnosis. PLoS Genet. 2011;7:e1002300.

26. Teschendorff AE, Menon U, Gentry-Maharaj A, Ramus SJ, Gayther SA, Apostolidou S, Jones A, Lechner M, Beck S, Jacobs IJ, Widschwendter M. An epigenetic signature in peripheral blood predicts active ovarian cancer. PLoS One. 2009;4:e8274.

27. Guintivano J, Aryee MJ, Kaminsky ZA. A cell epigenotype specific model for the correction of brain cellular heterogeneity bias and its application to age brain region and major depression. Epigenetics. 2013;8:290-302.

28. Houseman EA, Accomando WP, Koestler DC, Christensen BC, Marsit CJ, Nelson $\mathrm{HH}$, Wiencke JK, Kelsey KT. DNA methylation arrays as surrogate measures of cell mixture distribution. BMC Bioinformatics. 2012;13:86.

29. Gagnon-Bartsch JA, Speed TP. Using control genes to correct for unwanted variation in microarray data. Biostatistics. 2012;13:539-52.

30. Zou J, Lippert C, Heckerman D, Aryee M, Listgarten J. Epigenome-wide association studies without the need for cell-type composition. Nat Methods. 2014:11:309-11.

31. Rahmani E, Zaitlen N, Baran Y, Eng C, Hu D, Galanter J, Oh S, Burchard EG, Eskin E, Zou J, Halperin E. Sparse PCA corrects for cell type heterogeneity in epigenome-wide association studies. Nat Methods. 2016;13:443-5.

32. Caye K, Jumentier B, Lepeule J, Francois O. LFMM 2: Fast and Accurate Inference of Gene-Environment Associations in Genome-Wide Studies. Mol Biol Evol. 2019;36:852-60.

33. Patterson HD, Thompson R. Recovery of inter-block information when block sizes are unequal. Biometrika. 1971;58:545.

34. Yang J, Benyamin B, McEvoy BP, Gordon S, Henders AK, Nyholt DR, Madden PA, Heath AC, Martin NG, Montgomery GW, et al. Common SNPs explain a large proportion of the heritability for human height. Nat Genet. 2010;42:565-9.

35. Yang J, Lee SH, Goddard ME, Visscher PM. GCTA: a tool for genome-wide complex trait analysis. Am J Hum Genet. 2011;88:76-82.

36. Henderson CR. Best linear unbiased estimation and prediction under a selection model. Biometrics. 1975;31:423-47.

37. Zhou X, Stephens M. Genome-wide efficient mixed-model analysis for association studies. Nat Genet. 2012:44:821-4.

38. Kang HM, Sul JH, Service SK, Zaitlen NA, Kong SY, Freimer NB, Sabatti C, Eskin E. Variance component model to account for sample structure in genome-wide association studies. Nat Genet. 2010;42:348-54.

39. Yang J, Zaitlen NA, Goddard ME, Visscher PM, Price AL. Advantages and pitfalls in the application of mixed-model association methods. Nat Genet. 2014;46:100-6.

40. Lippert C, Listgarten J, Liu Y, Kadie CM, Davidson Rl, Heckerman D. FaST linear mixed models for genome-wide association studies. Nat Methods. 2011;8:833-5.

41. Meissner A, Mikkelsen TS, Gu H, Wernig M, Hanna J, Sivachenko A, Zhang X, Bernstein BE, Nusbaum C, Jaffe DB, et al. Genome-scale DNA methylation maps of pluripotent and differentiated cells. Nature. 2008;454:766-70.

42. Laurent L, Wong E, Li G, Huynh T, Tsirigos A, Ong CT, Low HM, Kin Sung KW, Rigoutsos I, Loring J, Wei CL. Dynamic changes in the human methylome during differentiation. Genome Res. 2010;20:320-31.

43. McGregor K, Bernatsky S, Colmegna I, Hudson M, Pastinen T, Labbe A, Greenwood CM. An evaluation of methods correcting for cell-type heterogeneity in DNA methylation studies. Genome Biol. 2016;17:84.

44. Starr JM, Deary IJ. Sex differences in blood cell counts in the Lothian Birth Cohort 1921 between 79 and 87 years. Maturitas. 2011;69:373-6.

45. Devlin B, Roeder K. Genomic control for association studies. Biometrics. 1999;55:997-1004

46. Goring HH, Curran JE, Johnson MP, Dyer TD, Charlesworth J, Cole SA, Jowett $J B$, Abraham LJ, Rainwater DL, Comuzzie AG, et al. Discovery of expression QTLs using large-scale transcriptional profiling in human lymphocytes. Nat Genet. 2007:39:1208-16.

47. Charlesworth JC, Curran JE, Johnson MP, Goring HH, Dyer TD, Diego VP, Kent JW Jr, Mahaney MC, Almasy L, MacCluer JW, et al. Transcriptomic epidemiology of smoking: the effect of smoking on gene expression in lymphocytes. BMC Med Genet. 2010;3:29.

48. Kent JW Jr, Goring HH, Charlesworth JC, Drigalenko E, Diego VP, Curran JE, Johnson MP, Dyer TD, Cole SA, Jowett JB, et al. Genotypexage interaction in human transcriptional ageing. Mech Ageing Dev. 2012;133:581-90. 
49. Mendelson MM, Marioni RE, Joehanes R, Liu C, Hedman AK, Aslibekyan S, Demerath EW, Guan W, Zhi D, Yao C, et al. Association of body mass index with DNA methylation and gene expression in blood cells and relations to cardiometabolic disease: a Mendelian randomization approach. PLoS Med. 2017;14:e1002215

50. Gao X, Jia M, Zhang Y, Breitling LP, Brenner H. DNA methylation changes of whole blood cells in response to active smoking exposure in adults: a systematic review of DNA methylation studies. Clin Epigenetics. 2015;7:113.

51. Shenker NS, Polidoro S, van Veldhoven K, Sacerdote C, Ricceri F, Birrell MA, Belvisi MG, Brown R, Vineis P, Flanagan JM. Epigenome-wide association study in the European Prospective Investigation into Cancer and Nutrition (EPIC-Turin) identifies novel genetic loci associated with smoking. Hum Mol Genet. 2013;22:843-51.

52. Listgarten J, Lippert C, Kadie CM, Davidson RI, Eskin E, Heckerman D. Improved linear mixed models for genome-wide association studies. Nat Methods. 2012;9:525-6.

53. Peters MJ, Joehanes R, Pilling LC, Schurmann C, Conneely KN, Powell J, Reinmaa E, Sutphin GL, Zhernakova A, Schramm K, et al. The transcriptional landscape of age in human peripheral blood. Nat Commun. 2015;6:8570

54. Zhang Q, Vallerga C, Walker R, Lin T, Henders A, Montgomery G, He J, Fan D, Fowdar J, Kennedy M, et al. Improved prediction of chronological age from DNA methylation limits it as a biomarker of ageing. bioRxiv. 2018; https://doi.org/10.1101/327890.

55. Hayeck TJ, Zaitlen NA, Loh PR, Vilhjalmsson B, Pollack S, Gusev A, Yang J, Chen GB, Goddard ME, Visscher PM, et al. Mixed model with correction for case-control ascertainment increases association power. Am J Hum Genet. 2015;96:720-30.

56. Weissbrod O, Lippert C, Geiger D, Heckerman D. Accurate liability estimation improves power in ascertained case-control studies. Nat Methods. 2015;12:332-4.

57. Chen H, Wang C, Conomos MP, Stilp AM, Li Z, Sofer T, Szpiro AA, Chen W, Brehm JM, Celedon JC, et al. Control for population structure and relatedness for binary traits in genetic association studies via logistic mixed models. Am J Hum Genet. 2016;98:653-66.

58. Zhou W, Nielsen JB, Fritsche LG, Dey R, Gabrielsen ME, Wolford BN, LeFaive J, VandeHaar P, Gagliano SA, Gifford A, et al. Efficiently controlling for casecontrol imbalance and sample relatedness in large-scale genetic association studies. Nat Genet. 2018;50:1335-41.

59. Deary IJ, Gow AJ, Pattie A, Starr JM. Cohort profile: the Lothian Birth Cohorts of 1921 and 1936. Int J Epidemiol. 2012;41:1576-84.

60. Taylor AM, Pattie A, Deary IJ. Cohort profile update: the Lothian Birth Cohorts of 1921 and 1936. Int J Epidemiol. 2018;47:1042-1042r.

61. Price ME, Cotton AM, Lam LL, Farre P, Emberly E, Brown CJ, Robinson WP, Kobor MS. Additional annotation enhances potential for biologicallyrelevant analysis of the Illumina Infinium HumanMethylation450 BeadChip array. Epigenetics Chromatin. 2013;6:4.

62. Deary IJ, Whiteman MC, Starr JM, Whalley L, Fox HC. The impact of childhood intelligence on later life: following up the Scottish mental surveys of 1932 and 1947. J Pers Soc Psychol. 2004;86:130-47.

63. Deary IJ, Gow AJ, Taylor MD, Corley J, Brett C, Wilson V, Campbell H, Whalley LJ, Visscher PM, Porteous DJ, Starr JM. The Lothian Birth Cohort 1936: a study to examine influences on cognitive ageing from age 11 to age 70 and beyond. BMC Geriatr. 2007;7:28.

64. Marioni RE, Shah S, McRae AF, Chen BH, Colicino E, Harris SE, Gibson J, Henders AK, Redmond P, Cox SR, et al: DNA methylation age of blood predicts all-cause mortality in later life. EMBL-EBI 2015, https://www.ebi.ac. uk/ega/studies/EGAS00001000910. [cited 25 May 2019]

65. Goring HH, Curran JE, Johnson MP, Dyer TD, Charlesworth J, Cole SA, Jowett JB, Abraham $\sqcup$, Rainwater DL, Comuzzie AG, et al. Discovery of expression QTLs using large-scale transcriptional profiling in human lymphocytes EMBL-EBI. 2008; https://www.ebi.ac.uk/arrayexpress/experiments/E-TABM305/. [cited 25 May 2019]

66. Zhang F, Chen W, Zhu Z, Zhang Q, Nabais MF, Qi T, Deary IJ, Wray NR, Visscher PM, McRae AF, Yang J. OSCA: a tool for omic-data-based complex trait analysis. Source Code Zenodo Repository. 2019. https://doi.org/10. 5281/zenodo.2658802. [cited 25 May 2019]

\section{Publisher's Note}

Springer Nature remains neutral with regard to jurisdictional claims in published maps and institutional affiliations.

Ready to submit your research? Choose BMC and benefit from:

- fast, convenient online submission

- thorough peer review by experienced researchers in your field

- rapid publication on acceptance

- support for research data, including large and complex data types

- gold Open Access which fosters wider collaboration and increased citations

- maximum visibility for your research: over $100 \mathrm{M}$ website views per year

At BMC, research is always in progress.

Learn more biomedcentral.com/submissions 